\title{
Synthetic Aperture Radar Ship Detection Using Haar-like Features
}

\author{
C. P. Schwegmann, W. Kleynhans and B. P. Salmon
}

\begin{abstract}
The detection of ships at sea is a complex task made more so by adverse weather conditions, lack of night visibility and large areas of concern. Synthetic Aperture Radar imagery with large swaths can provide the needed coverage at a reduced resolution. The development of ship detection methods that can effectively detect ships despite the reduced image resolution is an important area of research. A novel ship detection method is introduced that makes use of a standard Constant False Alarm Rate prescreening step followed by a cascade classifier ship discriminator. Ships are identified using Haar-like features using AdaBoost training on the classifier with an accuracy of $\mathbf{8 9 . 3 8 \%}$ and false alarm rate of $1.47 \times 10^{-8}$ across a large swath Sentinel-1 and RADARSAT-2 newly created SAR dataset.
\end{abstract}

Keywords-Synthetic aperture radar, Image processing, Pattern recognition, Marine technology

\section{INTRODUCTION}

$\mathbf{S}$ YNTHETIC Aperture Radar (SAR) surveillance is an integral part of large ocean area monitoring for Maritime Domain Awareness (MDA) [1]. Ship detection in SAR imagery has been a topic of research for a number of years [2] but access to SAR data has historically been problematic due to high data prices. Sentinel-1 has provided the world access to SAR imagery for free which enables countries to develop new MDA capabilities. Sentinel-1 imagery comes in various formats which include high resolution Single Look Complex (SLC) Interferometric Wide (IW) swath and intensity-only Extra Wide swath (EW) SAR imagery. The high spatial resolution of IW imagery makes it ideal for monitoring small areas near shorelines and inland surveillance whilst EW imagery is used to monitor larger areas such as Exclusive Economic Zones (EEZ), deeper ocean and icy regions.

Current ship detection research is focused on using high resolution IW Sentinel-1 imagery [3], [4], [5], instead of EW imagery [5], [6]. IW high resolution imagery allows for precise monitoring of small areas such as ports and shoreline areas. Additionally, the higher resolution data can facilitate algorithms that cannot necessarily be created using medium resolution imagery such as ship-type classification. However, the impracticality of monitoring large areas using only high resolution SAR imagery demands effective capability be developed to detect ships in low-to-medium resolution SAR imagery.

Ship detection in SAR intensity imagery is split into two steps after preprocessing, namely ship prescreening and ship discrimination [1], [6], [2]. Preprocessing concerns itself with the geocoding and removal of land (and sometimes SAR image errors such as ghosting) [2]. Most research in SAR ship detection, however, is focused on the final two steps. These

C. P. Schwegmann and W. Kleynhans are with the Department of Electrical, Electronic and Computer Engineering, University of Pretoria as well as the Remote Sensing Research Unit, Meraka Institute, CSIR, Pretoria, South Africa. Email: cschwegmann@csir.co.za. Telephone: +27 128413207.

B.P Salmon is with the School of Engineering and ICT, University of Tasmania, Australia. steps are the part of the process which run through a SAR image and prescreen pixels to determine if they are reflective and then discriminate among reflective pixels to only highlight ships. In many research papers these two steps are used separately [1], [6] but some research has shown it can also be approximated using a finely tuned single prescreening step [5]. Almost exclusively in ship detection studies the prescreening step is configured to stringently remove as many false alarms as possible [5], [6] which reduces the responsibility of the ship discrimination step. The difficulty this is that stringent removal of false alarms in the early stages increases the likelihood of not detecting ships later on (false alarms versus true positive trade-off). Depending on the requirements of the ship detection process and operator, this may not be a desirable result. Depending on the prescreening and discrimination method used, a significant increase in computational complexity can also occur [6], especially for large SAR imagery.

This paper introduces a new ship detection method which combines an unconventional low-threshold presecreening step with a novel ship discrimination step that extracts ship-like features and presents these to a classifier tailored to effectively identify SAR ships from false alarms. These unique features are scalable, rapidly calculable and are descriptive enough to highlight ships. Thanks to the carefully considered prescreening step, these features are only extracted for likely objects during detection, thereby improving the methods efficiency on SAR imagery. The combination of these adaptable features and versatile machine intelligence-based ship discrimination allows the proposed method to perform well across a large swath multi-sensor, multi-resolution newly created SAR ship dataset.

\section{Data Description And Study ARea}

The large swath SAR dataset was created to evaluate ship detection, discrimination and analysis methods in a bid to emulate operational ship detection scenarios with a SAR dataset. The dataset is highly diverse containing ships of various sizes, resolutions and acquisitions scenarios chosen in order to evaluate the adaptability of the proposed method and that of future methods. The dataset consists of 46 SAR images covering approximately $80 \%$ of South Africa's EEZ across: two sensors (RADARSAT-2 and Sentinel-1A); three resolutions (SCNA $25 \mathrm{~m} \times 25 \mathrm{~m}$, EW GRDH $25 \mathrm{~m} \times 25 \mathrm{~m}$ and EW GRDM $40 \mathrm{~m} \times 40 \mathrm{~m}$ ); and four polarizations $(\mathrm{HH}$, $\mathrm{HV}, \mathrm{VV}, \mathrm{VH})$. There are 1596 ships (positive samples) across all 46 images, extracted as $21 \times 21$ pixel sub-images for this experiment (accommodating a maximum possible ship size of 20 pixels long). An additional $319221 \times 21$ sub-images with no ships within them were used as negative samples.

\section{Methodology}

The method proposed has three parts. The low-threshold Constant False Alarm Rate (CFAR) prescreening method identifies likely ship candidates. This is followed by novel Haar-like feature extraction which is then fed to an adaptable cascade classifier to discriminate ships from non-ships. The details of these three steps are discussed next. 


\section{A. CA-CFAR prescreening}

CFAR is a ship prescreening method that uses local thresholding to detects unusually bright pixels (ships) via a sliding window configuration seen in Fig. 1. A Cell-Avaraging CFAR (CA-CFAR) compares the center region of interest's (ROI) window mean $\left(\mu_{\mathrm{ROI}}\right)$ to the background clutter's window mean $\left(\mu_{\mathrm{C}}\right)$ with a guard window to prevent ROI value corruption of the clutter mean. As the window moves across the image, ROI pixels which are $T_{\mathrm{C}}$ times brighter than $\mu_{\mathrm{C}}$ are marked as bright pixels in an output binary image $\mathbf{J}$. The threshold (or depending on the type of CFAR method the probability of false alarm $P_{\mathrm{fa}}$ ) can either be a fixed constant or two-dimensional plane which can be varied across a SAR image [1].

Assuming an input SAR intensity image $\mathbf{I}$ and binary output image $\mathbf{J}$ with dimensions $X \times Y$ where $x=\{0, \ldots, X-1\}$, $y=\{0, \ldots, Y-1\}$ and $x, y \in \mathbb{N}$ such that $\mathbf{I}$ and $\mathbf{J}$ can be defined as in [1] by

$$
\begin{aligned}
\mathbf{I} & =\left\{\{I(x, y)\}_{x=0}^{x=X-1}\right\}_{y=0}^{y=Y-1} \\
& =\left[\begin{array}{ccc}
I(0,0) & \cdots & I(0, Y-1) \\
I(1,0) & \cdots & I(1, Y-1) \\
\vdots & \ddots & \vdots \\
I(X-1,0) & \cdots & I(X-1, Y-1)
\end{array}\right], \\
\mathbf{J}\left(\mathbf{I}, T_{\mathrm{C}}\right) & =\left\{\left\{J\left(\mathbf{I}, x, y, T_{\mathrm{C}}\right)\right\}_{x=0}^{x=X-1}\right\}_{y=0}^{y=Y-1}
\end{aligned}
$$

Where $T_{\mathrm{C}}$ is known as the CA-CFAR threshold and is inversely proportional to the number of false alarm pixels permissible. The CA-CFAR binary pixel $J\left(\mathbf{I}, x, y, T_{\mathrm{C}}\right)$ and mean (power) ratio $\mu_{\text {ratio }}(x, y)$ are calculated with

$$
\begin{aligned}
J\left(\mathbf{I}, x, y, T_{\mathrm{C}}\right) & =\left\{\begin{array}{ll}
\text { true } & \text { if } \mu_{\text {ratio }}(x, y)>T_{\mathrm{C}} \\
\text { false } & \text { otherwise }
\end{array},\right. \\
\mu_{\text {ratio }}(x, y) & =\frac{\mu_{\mathrm{ROI}}(x, y)}{\mu_{\mathrm{C}}(x, y)} .
\end{aligned}
$$

SAR ship sub-images are extracted where $J(x, y)=$ true and processed in the next stage to extract these ship-like features. Using this prescreening method as presented here reduces the number of sub-images presented to the classifier by at least an order of magnitude whilst still presenting all the possible ships in an image due to the low-threshold chosen.

\section{B. Haar-like feature extraction}

The process of detecting all ships in an image can be accomplished if it is assumed that ships are brighter than their surroundings and a low enough threshold $\left(T_{\mathrm{C}}=1.0\right)$ CFAR will detect all ships in an image [1], [2]. To remove the many accompanying false alarms we propose to use special ship-like features called Haar-like features. These features have a number of directly relevant advantages for SAR ship discrimination as they are scalable, rapidly calculable and also reduce the inclass variance whilst increasing the out-of-class variance [7], [8]. Furthermore, using variants that are rotated, the features are simultaneously scale and rotation invariant [7], [8]. These features, therefore, aid in ship description in the current dataset but are extensible to allow the proposed discrimination method to work with future SAR data. An example of how the features look, scale and rotate to describe ships effectively is shown in Fig. 2. Haar-like features come in three types (edge, line and center) and two variations (upright [7] and rotated [8]).

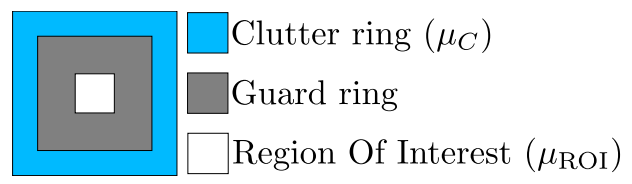

Fig. 1: The CA-CFAR window configuration where the mean pixel value inside the clutter and ROI rings are calculated as $\mu_{\mathrm{C}}$ and $\mu_{\mathrm{ROI}}$, respectively. The clutter ring is used to represent each pixels mean ocean backscatter or clutter level. The guard ring is in place to prevent corruption of the clutter mean by objects larger than the ROI.

A single Haar-like feature is simply the normalised difference between two (or more) scalable areas in a SAR ship sub-image. To aid in rapid calculation of these features, the concept of integral images is introduced [7]. An integral image II is the integral result of $\mathbf{I}$ and is calculated as the summation of all pixels above and to the left of a pixel at $I(x, y)$, inclusive. More specifically, $I I(x, y)=\sum_{x^{\prime} \leq x} \sum_{y^{\prime} \leq y} I\left(x^{\prime}, y^{\prime}\right)$. The advantage of using an integral image to calculate sums is that a single pass over the image is all that is required to calculate any integral image value and subsequent values only require reference lookups to II. Assuming $I I(-1, y)=I I(x,-1)=$ 0 [8], the integral image value at pixel $(x, y)$ is calculated using only four references to II such that

$$
\begin{aligned}
I I(x, y) & =I I(x, y-1)+I I(x-1, y)+I(x, y) \\
& -I I(x-1, y-1) .
\end{aligned}
$$

To calculate a single two-part Haar-like feature, $f_{j}$ we make use of the integral image to calculate the value over the two template areas using only six integral image references. Assuming two template rectangles $r_{1}$ and $r_{2}$ with heights $h_{1}$ and $h_{2}$ and widths $w_{1}$ and $w_{2}$, a two-part upright Haar-like feature $f_{j}$ is calculated as

$$
\begin{aligned}
f_{j} & =\frac{\Omega\left(r_{1}\right)-\Omega\left(r_{2}\right)}{w_{1} \times h_{1}+w_{2} \times h_{2}}, \\
\Omega(r) & =I I(x-1, y-1)+I I(x+w-1, y+h-1) \\
& -I I(x-1, y+h-1)-I I(x+w-1, y-1) .
\end{aligned}
$$

where $f_{j}$ is normalised by the area of the two templates so that features of different scales are weighted equally and $\Omega(r)$ is the pixel sums for $r_{1}$ and $r_{2}$, respectively. Due to two common points, $f_{j}$ only requires six references to II, irrespective of where or how large the feature is within the sub-image. The above only applies to upright features, but can be extended to include rotated features using a rotated integral image [8] thereby improving rotation invariance. For an illustrative example of how to calculate a single upright two part Haar-like feature see Fig. 3. Once these features are extracted they are presented to a trained classifier created using AdaBoost, discussed next.

\section{Cascade classifier creation using AdaBoost}

A single $21 \times 21$ SAR ship sub-image can contain up to 111160 (upright) or 155060 (upright+rotated) Haar-like features. Typically, this would be too many features for conventional classifiers to successfully train on due to the curse of dimensionality. To effectively select the most descriptive Haarlike features and create a classifier to discriminate ships the Adaptive Boosting (AdaBoost) method is chosen [7], [8], [9]. AdaBoost does this using three main concepts: weak learners or feature thresholds; strong learners which are weighted combinations of weak learners; and sample importance to identify which samples are most difficult to classify. By intelligently 


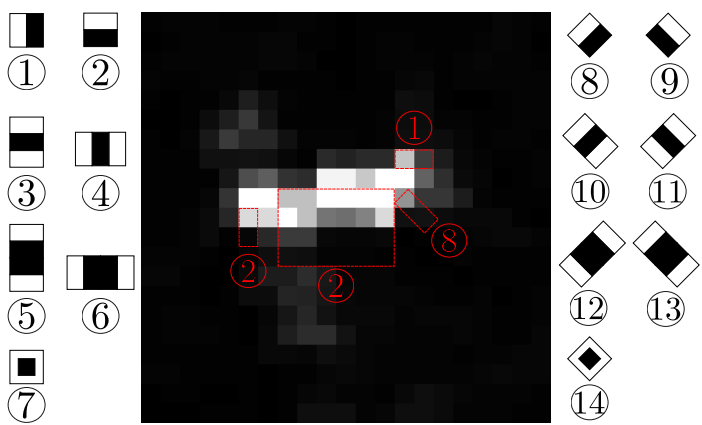

Fig. 2: A $21 \times 21$ RADARSAT-2 SAR intensity sub-image containing a ship with the upright [7] and rotated [8] Haar-like feature templates. Each ship can be described by a combination of these feature templates, at various scales and positions in the image.

ordering the strong learners in a cascade classifier, AdaBoost also ensures that only the most likely ship candidates reach the final stages where the stages are most complex thereby improving classification computational efficiency [7] (easy-todiscriminate samples are quickly accepted/discarded in the first stages). An example of the proposed classifier using strong and weak learners is shown in Fig. 4.

The AdaBoost creation of the cascade classifier proceeds over $L$ rounds where $l=1,2, \ldots, L$. For each round $S_{l}$ steps occur so that $s=1,2, \ldots, S_{l}$. Assuming $M$ samples (both ship and ocean) such that $m=1,2, \ldots, M$ with $N$ features such that $n=1,2, \ldots, N$ where each sample $x_{1}, x_{2}, \ldots, x_{M}$ is $x_{m}=\left\{f_{1}, f_{2}, \ldots, f_{N}\right\}$ and $f_{n}$ is the nth Haar-like feature. For each sample we assume a label $y_{m} \in\{-1,1\}$ for ocean and ships examples, respectively. Additionally, each sample $x_{m}$ has a sample importance distribution value $D_{s}(m)=$ $\{D(1), D(2), \ldots, D(M)\}$. For the first step $s=1$ all samples are weighted equally such that $D_{1}(m)=\frac{1}{M}$.

For each round $l$ a strong learner $H_{l}(x)$ is created that is a linear combination of weighted weak learners $h_{s}$. The simplest weak learner function that can be defined is a threshold $\theta_{n}$ of a single feature $f_{n}$ such that $h_{n}\left(x_{m}\right)=f_{n}>\theta_{n}$. This threshold is selected as the value that separates the $M$ samples with a fixed error less than 0.5 for each feature $n$. A single weak learners $h_{n}$ at $s$ ( $h_{s}$ for brevity) is selected from the set of all weak learners $\mathcal{H}$ at time step $s$ when it has the lowest error rate (at least $\epsilon_{n}<0.5$ ) and is assigned a weight $\alpha_{s}$ using

$$
\begin{aligned}
h_{s} & =\underset{h_{n} \in \mathcal{H}}{\arg \min } \sum_{m=1}^{M} D_{s}(m)\left[h_{n}\left(x_{m}\right) \neq y_{m}\right], \\
\alpha_{s} & =\frac{1}{2} \log \left(\frac{1+r_{s}}{1-r_{s}}\right), \\
r_{s} & =\sum_{m=1}^{M} D_{s}(m) h_{s}\left(x_{m}\right) y_{m} .
\end{aligned}
$$

Where $\mathcal{H}$ contains $N=111160$ or $N=155060$ weak learners depending if upright or upright and rotated Haarlike features are selected. The weighting factor, $\alpha_{s}$, is known as the weak learner importance. As shown in eq. (10), weak learners $h_{s}$ with error rates closer to 0.5 will have lower $\alpha_{s}$ values and hence their contribution to the strong learner $H_{l}(x)$ will be decreased because they provide less discrimination capability. By combining enough of these weak learners, it is mathematically proven that the resulting strong learner (and by extension cascade of strong learners) can be trained to a

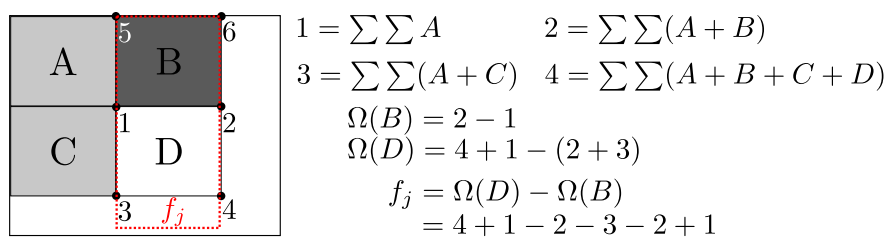

Fig. 3: Example calculation of an arbitrarily sized upright Haarlike feature within a SAR ship sub-image. Points 1 through 4 are calculated as the sum of pixels above and to the left of the point. These points are then used to calculate sums B and D (eq. (8)) using only two and four references, respectively. Finally, irrespective of size, feature $f_{j}$ can be calculated using eq. (7) with only six unique integral image references (normalisation not shown).

required accuracy and false alarm rate on the training data with a bounded generalization error [7], [8], [9].

The final action during step $s$ is the adjustment of the distribution $D_{s}(m)$ for the next step $s+1$. The distribution value at $m$ is increased for misclassified samples and decreased otherwise. The magnitude of this change is dependant on the previous distribution value of $D_{s}(m)$ and current weak learner importance $\alpha_{s}$ such that

$$
D_{s+1}(m)=\frac{D_{s}(m) \exp \left(-\alpha_{s} y_{m} h_{s}\left(x_{m}\right)\right)}{Z_{s}},
$$

where $Z_{s}$ is a normalisation factor chosen so that $D_{s+1}$ is a distribution. Adjusting the sample importance in this manner allows the AdaBoost procedure to hone in on features that can be thresholded such that $\epsilon<0.5$, even for difficult to classify samples when $s>1$. This is repeated $S_{l}$ times until the strong learner meets a specified minimum accuracy and false alarm rate.

Once $S_{l}$ steps have taken place using eq. (9), eq. (10) and eq. (12), a strong learner $H_{l}(x)$ can be defined as a linear combination of $S_{l}$ weak learners $h_{s}(x)$ and weak learner importances $\alpha_{s}$ such that

$$
H_{l}(x)=\operatorname{sign}\left(\sum_{s=1}^{S_{l}} \alpha_{s} h_{s}(x)\right)
$$

After $L$ rounds a cascade classifier is created containing $L$ strong learners $H_{l}(x)$ which in turn are composed of $S_{l}$ weak learners. The AdaBoost procedure reorders the cascade classifier so that, typically, $S=1$ for $l=1$ and $S \geq 1$ for $l \geq 1$. This configuration is an integral part of the proposed method because it allows for the removal of easy-to-identify false positives at the beginning of the cascade while delegating difficult sample discrimination (requiring more features) to the end, thereby improving computational efficiency. Finally, if a SAR sub-image is classified as a ship by $L$ cascaded strong learners it is deemed a ship.

\section{RESUlts AND Discussion}

\section{A. Experimental Parameters}

Experimental results are shown in Table. I, visually in Fig. 5 and graphed in Fig. 6 across a range of values. To properly assess the results in Table. I the three metrics need to be defined. Specifically, if we assume a truth table with TP true positives, TN true negatives, FP false positives and FN false negative, then Detection Accuracy (DA) can be defined as $D A=T P /(T P+F N)$, False Alarm Rate (FAR) as $F A R=F P /(F P+T N)$ and the Matthew Correlation 


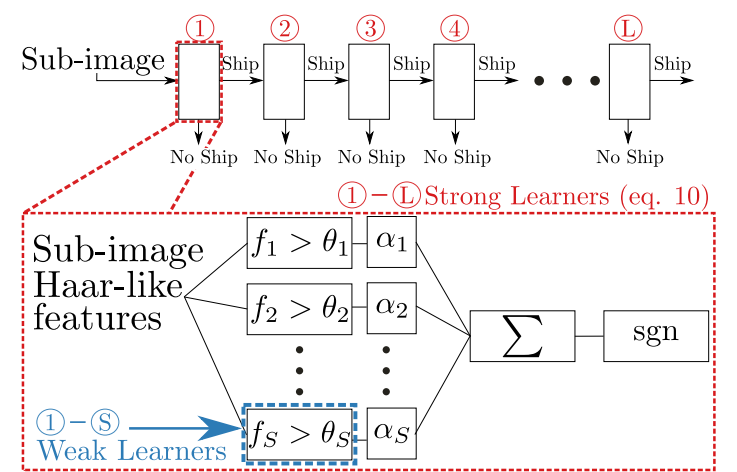

Fig. 4: A trained AdaBoost Haar-like feature cascade ship discriminator. A sub-image is fed into the cascade window and only the relevant Haar-like features are extracted per-stage. If a sub-image survives $L$ strong classifier stages then it is classified as a ship sample. Each strong classifier has $S$ weak classifiers within it where typically $S=1$ for the first stage and $S>1$ to improve computational efficiency.

Coefficient (MCC) [10] as

$$
\begin{aligned}
M C C= & \frac{T P \times T N-F P \times F N}{\sqrt{\mathcal{V}}} \\
\mathcal{V}= & (T P+F P) \times(T P+F N) \times \\
& (T N+F P) \times(T N+F N) .
\end{aligned}
$$

MCC values are $M C C \in\{-1.0,1.0\}$ which range from perfectly decorrelated $(-1.0)$ to perfectly correlated (1.0) with 0.0 indicating no correlation/random detections. The MCC is a particularly useful performance measure (metric) in this field as not only does it take equally into account all factors in the truth table but it is also unbiased by data with skewed classes [10] such as found in this study (many sea pixels versus few ships). The proposed method, CA-CFAR with a Haar-like feature classifier (CHAAR) (using full and uprightonly features) was compared to: a conventional CA-CFAR prescreening method; a stand-alone Haar-like feature classifier (HAAR) using full and upright-only features; a Modified Otsu's algorithm for ship detection [11]; and an H-dome transformation ship detector [12]. These were selected to identify the contributions of each component to performance as well as provide results against established and state-of-the-art large swath ship detection methods. Initial results indicated that the high dynamic range of SAR ships contributed to better performance so no contrast stretching on sub-images was performed, unlike [8]. Five fold cross validation was used with a data split of $80 \%$ for training and $20 \%$ for testing per fold which averages the test variance over the 5 repetitions. All CFAR prescreening methods used $1 \times 1,15 \times 15$ and $17 \times 17$ ROI, background and clutter windows, respectively, chosen based on the maximum window size of $21 \times 21$. The CFAR threshold was tested at high $T_{C}=5.0$ and low values $T_{C}=1.0$ to generate Table. I values but varied between these two to create the Receiver Operating Characteristic (ROC) curve shown in Fig. 6. The AdaBoost was given a target training performance of $95 \%$ detection accuracy (DA) and false alarm rate (FAR) of $0.1 \%$ in order to create the cascade classifier. The length of cascades $L$ was varied to obtain the ROC curves and for both the HAAR and CHAAR, the best performing cascade configurations had five strong learners $L=5$. The number of weak learners per stage $l=1, \ldots, 5$ was $S_{l}=\{1,3,1,2,94\}$ and $S_{l}=\{1,5,3,8,45\}$ for all (HAAR

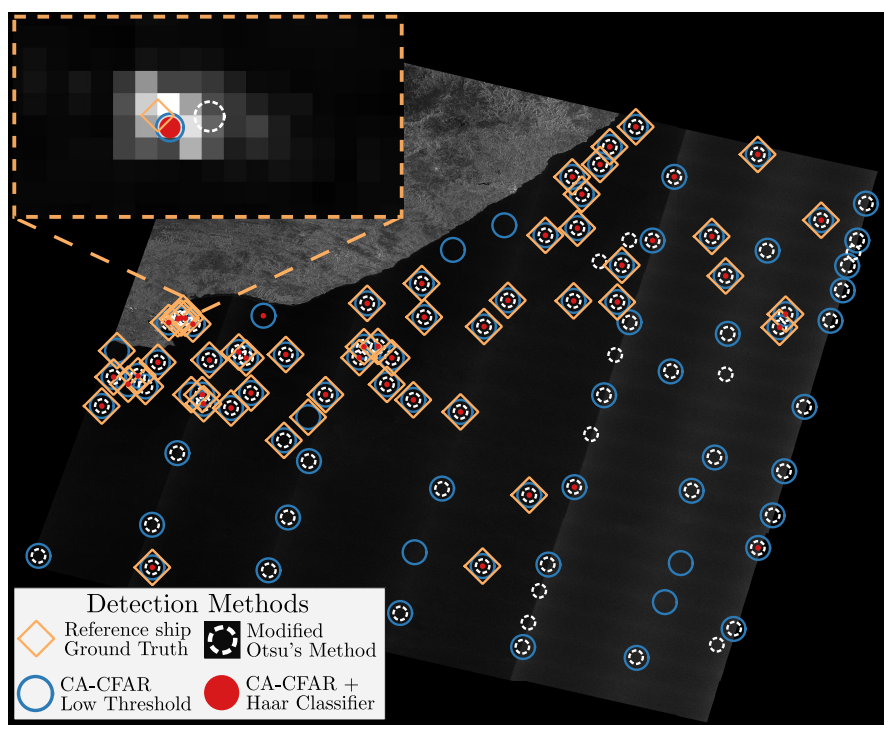

Fig. 5: Sentinel-1 GRDM VH image between Port Elizabeth and East London, South Africa ( $\left.33^{\circ} 57^{\prime} 29^{\prime \prime} \mathrm{S} 25^{\circ} 36^{\prime} 00^{\prime \prime} \mathrm{E}\right)$. The image shows 56 reference ships and the detection results of the CHAAR, CACFAR and Modified Otsu's method. The CHAAR has significantly lower number of false alarms (5 vs. 40 for the Mod. Otsu's) with only three missed detections. The cutout also shows the sub-pixel positional accuracy of the proposed ship detection method.

+ CHAAR) full and upright-only configurations, respectively. The Modified Otsu's ship detection method requires a minimum and maximum ship size set at 2 and 20 pixels determined by the maximum window size. The $\mathrm{H}$-dome transformation was tested using the recommended parameters values namely $\sigma_{L}=1.0, h=230$ and $d=5$ [12] for the table results but $h$ was varied between 200 and 255 for the ROC curve. The HAAR methods, in practice, use sliding windows to process large images and calculate a voting threshold $V$ to determine if multiple detection hits constitute a single object. The CHAAR method sets $V=1$ as each object is only presented once to the classifier.

\section{B. Results discussion}

Looking at Fig. 5 there are 56 ships in the image (diamonds). The CA-CFAR missed none, the Modified Otsu's missed two whereas the CHAAR method missed three. However, the CACFAR and Modified Otsu's detected 36 and 40 false alarms, respectively, whereas the CHAAR only detected 5. Also, the Modified Otsu's suffers with regards to positional accuracy with an average pixel error of 1.78 versus 0.68 for the CACFAR and CHAAR methods. This is likely due to the global threshold approach of the Modified Otsu's method compared to the local, adaptive threshold provided by the CA-CFAR and CHAAR methods.

A similar trend can be seen in Table. I and the ROC curve in Fig. 6. In practise, the three ships missed in Fig. 5 roughly translates to a $10 \%$ difference in DA compared to a lowthreshold CA-CFAR but only a $2-4 \%$ difference compared to other ship detection methods. While the CHAAR method has a lower DA than a conventional CA-CFAR, Modified Otsu's or $\mathrm{H}$-dome method, this is an acceptable trade-off considering the nearly two orders of magnitude improvement in FAR. While this might not satisfy every system requirement we feel the small trade off in DA is significantly off-set by the 
TABLE I: Mean DA, FAR and MCC results for the different systems tested across 5-fold cross validations with a total of 1596 ships in total tested. The values in brackets are the standard deviation and skewness values, respectively.

\begin{tabular}{llll}
\hline Method & DA & FAR & MCC \\
\hline CA-CFAR Low & $100.0(0$, N/A $)$ & $8.8016 \times 10^{-07}\left(5.8901 \times 10^{-07}, 1.14\right)$ & $0.41(0.01,-1.3)$ \\
CA-CFAR High & $65.69(2.6,-0.06)$ & $2.8262 \times 10^{-07}\left(1.8676 \times 10^{-07}, 1.19\right)$ & $0.44(0.02,-0.29)$ \\
HAAR Upright & $55.29(1.8,0.17)$ & $6.1761 \times 10^{-09}\left(3.4689 \times 10^{-09}, 0.85\right)$ & $0.72(0.01,0.99)$ \\
HAAR Full & $56.89(2.16,-1.33)$ & $9.1665 \times 10^{-09}\left(8.4399 \times 10^{-09}, 1.18\right)$ & $0.72(0.02,-0.59)$ \\
CHAAR Upright & $89.38(1.46,-0.13)$ & $1.4727 \times 10^{-08}\left(1.1691 \times 10^{-08}, 1.38\right)$ & $0.91(0.01,-1.29)$ \\
CHAAR Full & $81.83(2.79,0.05)$ & $3.4562 \times 10^{-08}\left(2.0646 \times 10^{-08}, 1.33\right)$ & $0.81(0.04,-0.52)$ \\
Mod. Otsu's & $90.00(1.29,-1.01)$ & $3.3585 \times 10^{-07}\left(2.0927 \times 10^{-07}, 1.06\right)$ & $0.54(0.03,-0.9)$ \\
H-dome & $93.82(0.27,-1.5)$ & $7.3376 \times 10^{-07}\left(4.8890 \times 10^{-07}, 1.12\right)$ & $0.42(0.01,-1.48)$ \\
\hline & & &
\end{tabular}

large difference in FAR. Objectively, the MCC value confirms this assessment as both CHAAR methods have higher MCC values than the other methods tested. The standard deviation and skewness lend a different aspect to this discussion. While the Modified Otsu's DA results have a slightly smaller spread compared to CHAAR-Upright (1.29 vs. 1.46), the CHAAR does have a more symetrical distribution of values about the mean DA compared to the Modified Otsu's ( -0.13 vs. -1.01$)$. Both methods are slightly negatively skewed, indicating DA results that are more consistently above the mean value. Looking towards Fig. 6 we see that the CHAAR-Upright has the best performance across a range of configurations with an AUC $=0.9257$. Interestingly, the HAAR and CHAAR curves are offset by a small difference likely due to the consistient DA provided by the CA-CFAR method employed in CHAAR.

Finally, the performance disparity between the two types of CHAAR methods needs to be discussed. The CHAAR-Upright method uses half the number of weak learners (45 vs 94) in its final stage and yet it outperforms the CHAAR-Full in every metric. This is likely due to the $39.49 \%$ larger feature space for the full set of Haar-like features and in turn causes a far more complicated final stage which appears to overfit the data slightly. This brings us to the conclusion that the full set of Haar-like features are not necessary to perform effective ship detection in SAR imagery.

\section{CONCLUSION}

The monitoring of large EEZ areas is a vital part of any country concerned with its MDA. SAR imagery, such as the free wide-swath imagery from Sentinel-1, can be effectively used to monitor such large ocean areas in an operational setting. However, in order to do so consistently, scientific investigation into current and new methods for ship detection needs to be evaluated constantly. Making use of multiple sources of SAR data in the creation of a broad ship detection dataset, including SAR imagery from different sensors and polarization, can help assess the consistency and adaptability of new methods.

This paper introduced a novel ship detection method tested against a large swath, medium resolution SAR dataset. By discerningly combining the high detection accuracy of lowthreshold CFAR prescreening with the persistent false alarm removal of a Haar-like AdaBoost trained cascade classifier, the proposed method had a DA of $89.38 \%$, FAR of $1.47 \times 10^{-8}$ and MCC of 0.91 , better than seven other methods tested. The combination of these unique components provides an adaptable, efficient and consistent ship detection method tested across a 46 SAR image dataset.

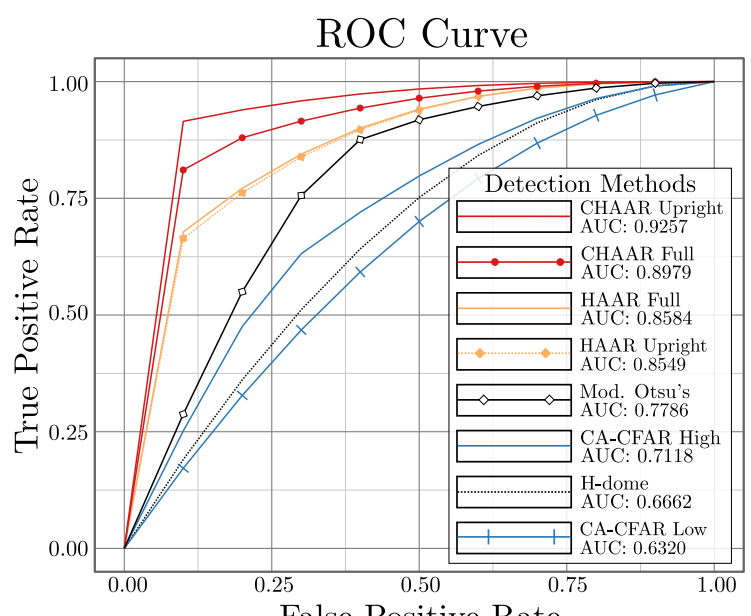

False Positive Rate

Fig. 6: Receiver Operating Characteristic (ROC) curves for the eight methods tested. The CHAAR-Upright method provides the highest AUC and has a small offset from the HAAR results likely due to the influence of the CA-CFAR prescreeninig. This False Positive Rate is scaled for display purposed compared to the results in Table. I.

\section{REFERENCES}

[1] C. Schwegmann, W. Kleynhans, and B. Salmon, "Manifold Adaptation for Constant False Alarm Rate Ship Detection in South African Oceans," IEEE Journal of Selected Topics in Applied Earth Observations and Remote Sensing, vol. 8, no. 7, pp. 3329-3337, 2015.

[2] D. J. Crisp, "The State-of-the-Art in Ship Detection in Synthetic Aperture Radar Imagery," Tech. Rep. DSTO-RR-0272, Australian Department of Defence, Edinburgh, Australia, 2004.

[3] J. Martin de Nicolas, P. Jarabo-Amores, N. del Rey-Maestre, P. Gomez del Hoyo, and J. L. Barcena-Humanes, "Robustness of a Generalized Gamma CFAR ship detector applied to TerraSAR-X and Sentinel-1 images," in EUROCON 2015 - International Conference on Computer as a Tool (EUROCON), IEEE, Sept 2015, pp. 1-6.

[4] D. Velotto, C. Bentes, B. Tings, and S. Lehner, "Comparison of sentinel1 and terrasar-x for ship detection," in Geoscience and Remote Sensing Symposium (IGARSS), 2015 IEEE International, July 2015, pp. 32823285.

[5] R. Pelich, N. Longepe, G. Mercier, G. Hajduch, and R. Garello, "Performance evaluation of Sentinel-1 data in SAR ship detection," in Geoscience and Remote Sensing Symposium (IGARSS), 2015 IEEE International, July 2015, pp. 2103-2106.

[6] P. Iervolino, R. Guida, and P. Whittaker, "A novel ship-detection technique for Sentinel-1 sar data," in Synthetic Aperture Radar (APSAR), 2015 IEEE 5th Asia-Pacific Conference on, 2015, pp. 797-801.

[7] P. Viola and M. Jones, "Rapid object detection using a boosted cascade of simple features," in Computer Vision and Pattern Recognition (CVPR), 2001 IEEE Computer Society Conference on, 2001, pp. I511-I-518 vol.1.

[8] R. Lienhart and J. Maydt, "An extended set of haar-like features for rapid object detection," in Image Processing (ICIP), 2002 IEEE International Conference on, 2002, pp. I-900-I-903 vol.1.

[9] R. E. Schapire, Nonlinear Estimation and Classification, pp. 149-171, Springer New York, New York, NY, 2003.

[10] B.W. Matthews, "Comparison of the predicted and observed secondary structure of t4 phage lysozyme," Biochimica et Biophysica Acta (BBA) - Protein Structure, vol. 405, no. 2, pp. 442-451, 1975.

[11] M. Messina, M. Greco, L. Fabbrini, and G. Pinelli, "Modified Otsu's algorithm: A new computationally efficient ship detection algorithm for SAR images," in Advances in Radar and Remote Sensing (TyWRRS), 2012 Tyrrhenian Workshop on, Sep 2012, pp. 262-266.

[12] C. P. Schwegmann, W. Kleynhans, B. P. Salmon, and L. Mdakane, "Ship detection in Sentinel-1 imagery using the H-dome transformation," in Geoscience and Remote Sensing Symposium, 2015. IGARSS 2015. IEEE International, 2015, pp. 3710-3714. 